University of Nebraska - Lincoln

DigitalCommons@University of Nebraska - Lincoln

\title{
Noninvasive Assessment of Cardiac Abnormalities in Experimental Autoimmune Myocarditis by Magnetic Resonance Microscopy Imaging in the Mouse
}

\author{
Chandirasegaran Massilamany \\ University of Nebraska-Lincoln, cmassilamany@unl.edu \\ Vahid Khalilzad-Sharghi \\ University of Nebraska-Lincoln, vahid0001@gmail.com \\ Arunakumar Gangaplara \\ University of Nebraska-Lincoln \\ David Steffen \\ University of Nebraska-Lincoln, dsteffen1@unl.edu \\ Shadi F. Othman \\ University of Nebraska - Lincoln, sothman2@unl.edu \\ Follow this and additional works at: https://digitalcommons.unl.edu/vbsjayreddy \\ I next page for additional authors \\ Part of the Biochemistry, Biophysics, and Structural Biology Commons, Cell and Developmental \\ Biology Commons, Veterinary Infectious Diseases Commons, Veterinary Microbiology and \\ Immunobiology Commons, and the Veterinary Physiology Commons
}

Massilamany, Chandirasegaran; Khalilzad-Sharghi, Vahid; Gangaplara, Arunakumar; Steffen, David; Othman, Shadi F.; and Reddy, Jay, "Noninvasive Assessment of Cardiac Abnormalities in Experimental Autoimmune Myocarditis by Magnetic Resonance Microscopy Imaging in the Mouse" (2016). Jay Reddy Publications. 29.

https://digitalcommons.unl.edu/vbsjayreddy/29

This Article is brought to you for free and open access by the Veterinary and Biomedical Sciences, Department of at DigitalCommons@University of Nebraska - Lincoln. It has been accepted for inclusion in Jay Reddy Publications by an authorized administrator of DigitalCommons@University of Nebraska - Lincoln. 


\section{Authors}

Chandirasegaran Massilamany, Vahid Khalilzad-Sharghi, Arunakumar Gangaplara, David Steffen, Shadi F. Othman, and Jay Reddy 


\title{
Video Article \\ Noninvasive Assessment of Cardiac Abnormalities in Experimental Autoimmune Myocarditis by Magnetic Resonance Microscopy Imaging in the Mouse
}

\author{
Chandirasegaran Massilamany ${ }^{1}$, Vahid Khalilzad-Sharghi ${ }^{2}$, Arunakumar Gangaplara ${ }^{1}$, David Steffen ${ }^{1}$, Shadi F. Othman ${ }^{2}$, Jay Reddy ${ }^{1}$ \\ ${ }^{1}$ School of Veterinary Medicine and Biomedical Sciences, University of Nebraska-Lincoln \\ ${ }^{2}$ Department of Biological Systems Engineering, University of Nebraska-Lincoln \\ * These authors contributed equally
}

Correspondence to: Shadi F. Othman at othman@unl.edu, Jay Reddy at jayreddy@unl.edu

URL: http://www.jove.com/video/51654

DOI: doi:10.3791/51654

Keywords: Medicine, Issue 88, Magnetic resonance microscopy, MRM, MRI, autoimmune myocarditis, mouse, noninvasive tool, heart, cardiac myosin heavy chain

Date Published: 6/20/2014

Citation: Massilamany, C., Khalilzad-Sharghi, V., Gangaplara, A., Steffen, D., Othman, S.F., Reddy, J. Noninvasive Assessment of Cardiac Abnormalities in Experimental Autoimmune Myocarditis by Magnetic Resonance Microscopy Imaging in the Mouse. J. Vis. Exp. (88), e51654, doi:10.3791/51654 (2014).

\section{Abstract}

Myocarditis is an inflammation of the myocardium, but only $\sim 10 \%$ of those affected show clinical manifestations of the disease. To study the immune events of myocardial injuries, various mouse models of myocarditis have been widely used. This study involved experimental autoimmune myocarditis (EAM) induced with cardiac myosin heavy chain (Myhc)- $\alpha$ 334-352 in A/J mice; the affected animals develop lymphocytic myocarditis but with no apparent clinical signs. In this model, the utility of magnetic resonance microscopy (MRM) as a non-invasive modality to determine the cardiac structural and functional changes in animals immunized with Myhc- $\alpha 334-352$ is shown. EAM and healthy mice were imaged using a $9.4 \mathrm{~T}(400 \mathrm{MHz}) 89 \mathrm{~mm}$ vertical core bore scanner equipped with a $4 \mathrm{~cm}$ millipede radio-frequency imaging probe and $100 \mathrm{G} / \mathrm{cm}$ triple axis gradients. Cardiac images were acquired from anesthetized animals using a gradient-echo-based cine pulse sequence and the animals were monitored by respiration and pulse oximetry. The analysis revealed an increase in the thickness of the ventricular wal in EAM mice, with a corresponding decrease in the interior diameter of ventricles, when compared with healthy mice. The data suggest that morphological and functional changes in the inflamed hearts can be non-invasively monitored by MRM in live animals. In conclusion, MRM offers an advantage of assessing the progression and regression of myocardial injuries in diseases caused by infectious agents, as well as response to therapies.

\section{Video Link}

The video component of this article can be found at http://www.jove.com/video/51654/

\section{Introduction}

Heart failure is the leading cause of deaths, and myocarditis is one predominant cause of heart failure in young adolescents ${ }^{1}$. Most patients affected with myocarditis remain asymptomatic and the disease is spontaneously resolved ${ }^{2}$. However, $10-20 \%$ of those affected can develop chronic disease, leading to dilated cardiomyopathy $(\mathrm{DCM})^{3}$. Various animal models have been developed to study the immune pathogenesis of myocarditis. The disease can be induced in myocarditis-susceptible $\mathrm{A} / \mathrm{J}$ and Balb/c mice by immunizing the animals with cardiac myosin heavy chain (Myhc)- $\alpha$ or its immunodominant peptide fragments or by infecting with pathogens like coxsackievirus B3 ${ }^{4-9}$. This study involves Myhca 334-352-induced myocarditis in A/J mice. Despite showing myocardial infiltrations, the myocarditis-affected animals appear clinically normal; diagnosis is based on histological evaluation of hearts for inflammation ${ }^{7}$ and echocardiography ${ }^{10}$.

Magnetic resonance microscopy (MRM) is a commonly used method to obtain cardiovascular imaging with high resolution three-dimensional planes, permitting assessment of functional details to the level of minute blood vessels (up to $10 \mu \mathrm{m}$ diameter), but this level of resolving power is not achievable with the routine magnetic resonance imaging (MRI) scanning procedure, in which, the resolution is generally obtained up to 1 $\mathrm{mm}^{11-14}$. MRM offers an advantage as it permits acquisition of high-resolution images and also to derive performance parameters in the early time points of disease process ${ }^{14}$. Clinically, MRM imaging has been extensively applied to study the functional parameters of diseased heart, lung or brain ${ }^{15-17}$. In this study, the use of an MRM technique as a non-invasive tool to ascertain cardiac abnormalities in $\mathrm{A} / \mathrm{J}$ mice affected with autoimmune myocarditis is shown. Specifically, the MRM imaging allows quantification of functional parameters such as left ventricular (LV) enddiastolic volume and ejection fraction (EF) with reasonable accuracy ${ }^{18}$. The definitions of respective parameters are: LV end diastolic volume, volume of blood in the left ventricle at the end of diastolic cycle, and ejection fraction, stroke volume/end-diastolic volume. The data analysis is performed using the freely available Segment software developed for processing DICOM-compliant cardiovascular images acquired by magnetic resonance scanners ${ }^{19}$. The data revealed an increase in thickness of the LV wall in the myocarditic animals, corresponding to a decrease in LV end-diastolic volume, stroke volume and ejection fraction, as compared with these functional parameters in healthy mice. 
Protocol

\section{ETHICS STATEMENT:}

All animal procedures were conducted in accordance with the guidelines for the Care and Use of Laboratory Animals and approved by the University of Nebraska-Lincoln, Lincoln, NE.

\section{Induction of Experimental Autoimmune Myocarditis}

1. Prepare the peptide solution by dissolving Myhc- $\alpha 334-352$ in $1 \mathrm{x}$ phosphate-buffered saline to a final concentration of $2 \mathrm{mg} / 1.5 \mathrm{ml}$.

2. Prepare the pertussis toxin (PT) by adding $1 \mathrm{ml}$ of sterile $1 \times$ PBS to a vial containing $50 \mu \mathrm{g}$ of lyophilized PT to obtain a stock concentration of $50 \mathrm{ng} / \mu \mathrm{l}$. Take $20 \mu \mathrm{l}$ of the stock into a sterile $1.5 \mathrm{ml}$ tube and add $980 \mu \mathrm{l}$ of sterile $1 \times$ PBS to obtain a working concentration of $1 \mathrm{ng} / \mu \mathrm{l}$.

3. Prepare complete Freund's adjuvant (CFA) by adding $40 \mathrm{mg}$ of Mycobacterium tuberculosis H37Rv (MTB) extract to $10 \mathrm{ml}$ of CFA to obtain a final concentration of $5 \mathrm{mg} / \mathrm{ml}$.

4. Prepare the peptide-CFA emulsion. NOTE: For EAM induction, the peptide-CFA emulsion was administered in $150 \mu \mathrm{l}$ containing $100 \mu \mathrm{g}$ of Myhc- $\alpha$ 334-352 per animal. For example, to immunize ten mice use $1.5 \mathrm{ml}$ of peptide-CFA emulsion containing $1 \mathrm{mg}$ of Myhc- $\alpha 334-352$.

1. To prepare $1.5 \mathrm{ml}$ of emulsion, aliquot $750 \mu \mathrm{l}$ of Myhc- $\alpha 334-352$ peptide solution in one $1.5 \mathrm{ml}$ tube, and CFA supplemented with MTB into another $1.5 \mathrm{ml}$ tube. Using a $3 \mathrm{ml}$ luer-lok syringe, draw the peptide solution first, followed by the CFA/MTB extract.

2. Attach the syringe to a 3-way stopcock and connect the other outlet of the stopcock to an empty $3 \mathrm{ml}$ syringe. Adjust the patency of the stopcock so the peptide-CFA mixture flows from one syringe to the other with reasonably good resistance.

3. Mix by pushing the contents from one syringe to the other repeatedly for $\sim 1$ min and then place the entire assembly on ice for $\sim 3$ min. Repeat this procedure a minimum of $10 x$.

4. Determine the consistency of the emulsion by carefully placing a tiny droplet on still water in a $100 \mathrm{ml}$ glass beaker. The droplet is not expected to disperse in water. If it does, continue mixing until the desired consistency is achieved.

5. Transfer the contents of emulsion from $3 \mathrm{ml}$ syringes into $1 \mathrm{ml}$ luer-lok syringes by replacing one of the two 3-ml syringes attached to the stopcock with the $1-\mathrm{ml}$ syringe, and attach a $271 / 2 \mathrm{G}$ needle to the $1 \mathrm{ml}$ syringe.

5. Inject $150 \mu \mathrm{l}$ of the peptide-CFA emulsion in split doses subcutaneously in the two inguinal regions of six-to-eight-week-old female $\mathrm{A} / \mathrm{J}$ mice $(\sim 75 \mu$ l each).

6. Administer $100 \mu \mathrm{l}$ of PT suspension ( $100 \mathrm{ng}$ ) intraperitoneally to each animal on day 0 and day 2 postimmunization.

7. Repeat the immunization procedure on day 7 by administering $150 \mu$ l of peptide-CFA emulsion in split doses subcutaneously into either side of the sternum ( $75 \mu \mathrm{l}$ each). Prepare this emulsion fresh as above. Then, on day 21 , subject the animals to MRM imaging, see step 3.

\section{Animal Handling}

1. Place each mouse in an anesthetic induction chamber containing $2 \%$ isoflurane-air mixture with a heating pad placed underneath to maintain warmth, and transfer the animal to a specially designed animal holder (Figure 1).

2. Immobilize the animal in the prone position on the animal holder so its snout fits into the nose cone to maintain anesthesia (Figure 1). Secure the head of the mouse with a bite-bar attached to the front teeth of the mouse.

3. Turn on the air blow heater with its outlet hose inserted in the scanner's vertical bore to maintain the animal's body temperature during the experiment.

4. Maintain anesthesia at 0.5 to $2 \%$ isoflurane with a flow rate of $2 \mathrm{ml} / \mathrm{min}$ during the entire imaging session. Confirm anesthesia using the toe pinch method, no movement expected.

5. Set up a pneumatic pillow sensor, mouse tail/ankle fiber optic pulse oximetry sensor, and rectal temperature probe to monitor respiration, heart rate and body temperature, respectively (Figure 1). Note: Cardiac gating is performed through Pulse Oximetry, which allows noninvasive monitoring of arterial blood oxygen saturation. The pulse oximetry sensor should be attached to the left ankle, and the foot should be secured with a thread loop and taped to maintain the ankle in the sensor. MRM imaging is achieved by gating the respiration and cardiac signals without the use of any contrast agents.

\section{Image Acquisition}

1. After preparing the animal (Figure 1), place the mouse in the center of the MRM scanner with the heart positioned in the center of the field of view (FOV), where magnetic field homogeneity is maximum. NOTE: A wide-bore $(89 \mathrm{~mm}) 9.4 \mathrm{~T}$ vertical-bore magnet equipped with triple axis gradients of $100 \mathrm{G} / \mathrm{cm}$ and a $4 \mathrm{~cm}$ radio-frequency (RF) imaging coil is to be used to obtain high-resolution three-dimensional (3D) images. Note: Be sure to non-magnetic supplies when using an MRI scanner.

2. Run the imaging interface and select "New Study" from the "Study Options" menu. Type "mtune" at the command bar and run it to pull up the "Tune GUI". Then select "Start Probe Tune" and click on "Autoscale" button. The Tune GUI will change to show the RFsignal. Use the remote tune/match knobs at the end of the coil to tune the RF coil to the proton resonance frequency ( $400 \mathrm{MHz})$. On the "Start" tab go to the "Prescan" page to run the frequency and power calibration by clicking on the related buttons. Hit the XYZ (quick) button on the 'Shim" tab of "Study' tab to pull up the shimming page. Go to the shim page, select all of the iterations, and hit the shim button to perform auto shimming.

3. Select a scout sequence from the "Study" tab of the imaging interface to localize the mouse heart. On the "Acquire" tab change the FOV to 35 $\mathrm{mm}^{2}$ and keep the machine's default settings. Click Start to run the sequence; adjust the position of the animal holder if the heart is not in the center of FOV, retune the RF coil and obtain the scout images again.

4. Click the "GEMS" sequence button on the "Study" tab then enter the acquisition parameters on the corresponding "Acquire" tab to obtain two orthogonal planes along the short axis and long axis of the heart ${ }^{20,21}$. NOTE: Typical acquisition parameters for a gradient-echo scan are: 
slice thickness, $1 \mathrm{~mm}$; repetition time (TR), $200 \mathrm{msec}$; echo time (TE), $2.67 \mathrm{msec}$ (minimum); flip angle, $25^{\circ}$; inplane matrix size, $128 \times 128$; FOV, $22 \mathrm{~mm}^{2}$; number of acquisitions, 4; and an approximate acquisition time of $1 \mathrm{~min}$ and $30 \mathrm{sec}$.

5. On the "Adv" tab select the "CINE" sequence to collect the pulse oximetry-gated short-axis cine MR images in order to measure the LV anatomic and functional parameters. Adjust the position and angle of the imaging slices based on the long axis view of the heart by mouse hovering and dragging. Enter the following imaging parameters on the "Acquire" tab to obtain the gradient echo cine sequence: TR, 500 msec; TE, 5 msec; FOV, 22 mm$^{2}$; acquisition matrix, 256 x 256; slice thickness, $1 \mathrm{~mm}$; number of acquisitions, 8; number of frames, 6; and an acquisition time of $\sim 17 \mathrm{~min}$. Click start to begin the acquisition.

6. Convert the acquired images to DICOM format using the "I/O" tab of the imaging software and transfer the corresponding files to the data center for processing.

7. At the end of imaging procedure, allow the mice to recover from anesthesia within the filter-top cages. Do not leave the mice unattended until they regain sufficient consciousness to maintain sternal recumbency and keep them for further studies as needed.

\section{Data Analysis of Cardiac Magnetic Resonance Microscopy Imaging}

1. Use Segment software to analyze the anatomic and functional parameters of the LV'. ${ }^{19}$ By loading the DICOM format cine images into the software using the "Open Image File(s)" submenu of the "File" menu. On the GUI select 'MRGE' as the imaging technique, 'Cine' as the image type and 'short-axis mid-ventricular' as the image view plane.

2. Specify the time frames to be used for end-diastole and end-systole through the "Set Current Timeframe to End Diastole" and "Set Current Timeframe to End Systole" submenus of the "Edit" menu respectively.

3. First click the "LV" command button and then the "ENDO" and "EPI" command buttons on the bottom-right panel to manually draw the left ventricle endocardium and epicardium respectively. Remove the papillary muscles by hitting the corresponding command button in order to increase the accuracy of the calculations.

4. Read the quantified LV parameters such as diastolic volume, systolic volume, stroke volume, and ejection fraction on the top-right panel. Click the "Misc" command button and then the "Measurement Caliper" command button to measure the LV parameters such as wall thickness, and ventricular diameter ${ }^{22}$

5. Click the "Save Both Image Stacks and Segmentation" submenu of the "File" menu to save the images, including the segmentations, in '.mat' format to reprocess the images as needed.

\section{Representative Results}

In this report, the utility of MRM technique as a non-invasive modality to determine the structural and functional changes in the hearts of animals affected with EAM is shown. Myocarditis was induced in A/J mice by immunizing the animals with Myhc- $\alpha 334-352$ in CFA ${ }^{7}$, and the animals were subjected to MRM experiments on day 21 post-immunization. The MRM imaging was performed on live animals under isoflurane anesthesia at $9.4 \mathrm{~T}$ (400 MHz for protons) using an $89 \mathrm{~mm}$ vertical-bore magnet equipped with triple axis gradients (maximum strength 100 $\mathrm{G} / \mathrm{cm}$ ). A scout image was acquired to locate and position the mouse heart in the center of the FOV, followed by axial images to obtain the long-axis 4-chamber view. The angle at which the heart was imaged for the 2-chamber view is shown in Figure 2A. Cardiac images were acquired using a $4 \mathrm{~cm}$ millipede RF imaging probe with a gradient-echo-based cine pulse sequence. Cardiac function measurements (imaging: LV wall thickness; output: LV end-diastolic volume and ejection fraction) were then analyzed using Segment software. Structural defects in the hearts of EAM-affected mice were evidenced by increase in LV thickness by approximately 1.5-fold $(p=0.018)$ (Figure 2B and Table 1), with corresponding decrease in LV end-diastolic volume [18.0 $\pm 4.2 \mu \mathrm{l}$ vs. $37.5 \pm 3.5 \mu \mathrm{l}$, Figure $2 \mathrm{C}(\mathrm{i}) ; \mathrm{p}=0.002]$ and ejection fraction [49.4 $\pm 2.3 \%$ vs. $71.5 \pm 6.0 \%, p=0.00066$; Figure $\mathbf{2 C}$ (ii)] as compared to healthy mice. As expected, histological evaluation of hearts from myocarditic, but not healthy, mice showed multifocal lymphocyte infiltrates, as we have demonstrated previously ${ }^{\prime}$; Figure 2D). The data suggest that morphological and functional changes in inflamed hearts can be non-invasively monitored by MRM in live animals.

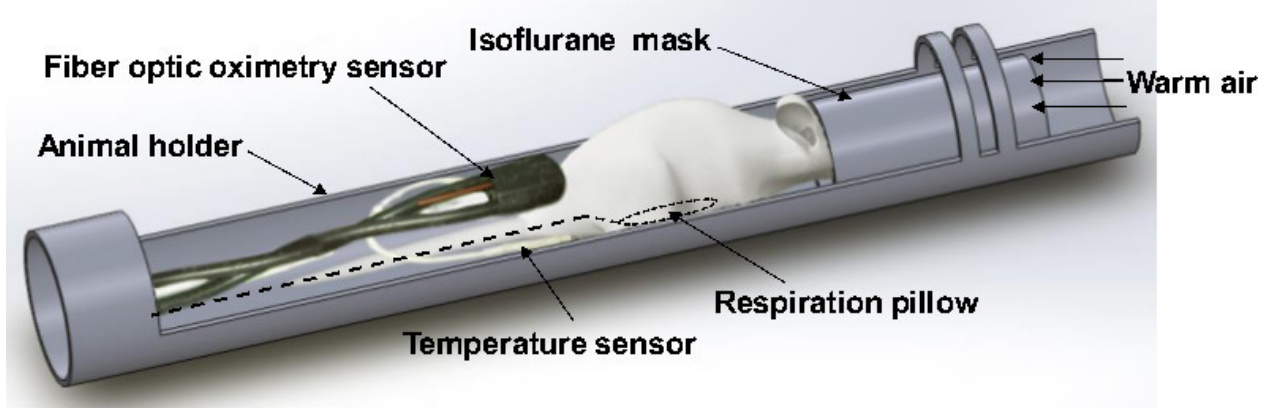

Figure 1. Animal preparation and positioning of probes for acquisition of MRM images from the mouse heart. To acquire images from the heart, the anesthetized mouse is placed in the animal holder specially designed for MRM imaging and connected to the air blow heater to maintain the body temperature. Under continuous anesthesia, the animal is immobilized in the prone position. A pneumatic pillow, fiber optic oximetry sensor and temperature sensor are set up to monitor respiration, pulse and body temperature, respectively, until MRM acquisition of cardiac images is complete. Please click here to view a larger version of this figure. 
(A) Position of MRM slicing
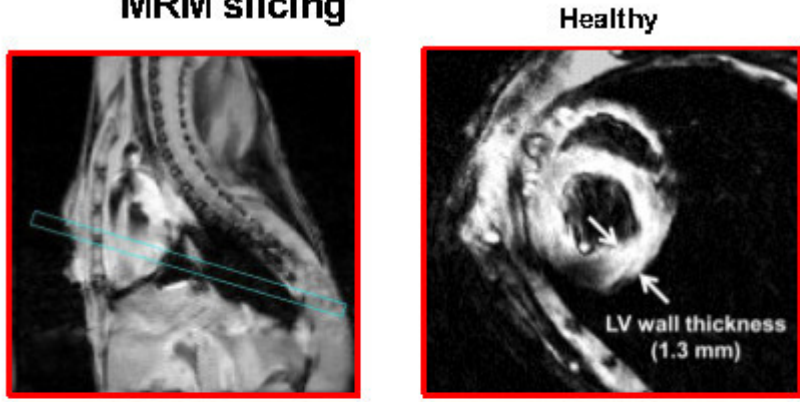

(B) MRM imaging

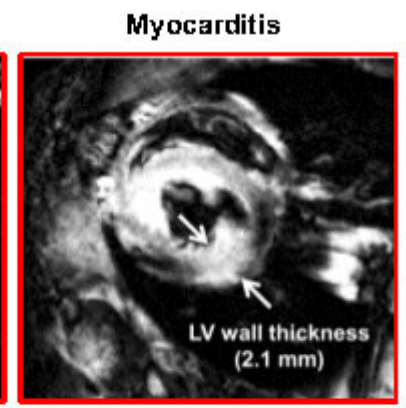

(C) Cardiac output

\section{(i) End diastolic (ii) Ejection fraction} volume

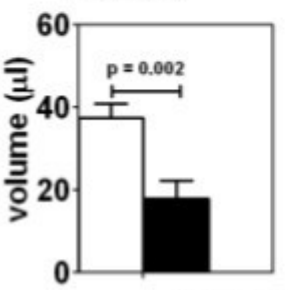

$\square$ Healthy
EAM

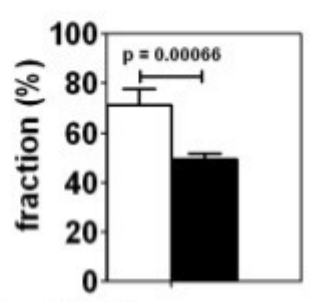

(D) Histology

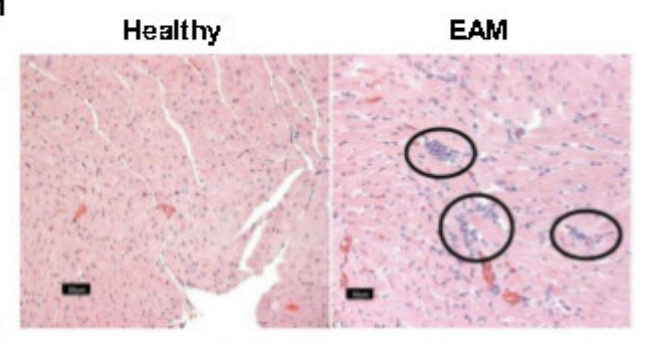

Figure 2. MRM imaging of mice affected with autoimmune myocarditis reveals cardiac abnormalities. Myocarditis was induced in $\mathrm{A} / \mathrm{J}$ mice by immunizing the animals with Myhc- $\alpha 334-352$ in CFA. The animals were subjected to MRM imaging on day 21 postimmunization to evaluate cardiac abnormalities. (A) Position of MRM slicing. The angle at which the heart was sliced for image acquisition is shown. (B) MRM imaging. Short-axis slices of heart were captured using echo-based cine pulse sequence in eight time frames with a TR of 500 msec (TE, 5 msec; flip angle, $20^{\circ}$; number of acquisitions, 4; acquisition matrix, $256 \times 256$ ) [arrows: LV wall thickness]. (C) Cardiac output. Cardiac output was measured based on (i) LV end-diastolic volume and (ii) ejection fraction in healthy and myocarditic mice using quantitative medical image analysis with Segment software. Mean SEM values for a group of mice are shown ( $n=2$ to 5 per group). (D) Histology. Hearts from the above treatment groups were evaluated for inflammation by hematoxylin and eosin staining. Circles: multifocal lymphocytic infiltrations. Please click here to view a larger version of this figure.

\begin{tabular}{|l|l|l|}
\hline Animals & Healthy $(\mathrm{n}=3)$ & EAM $(\mathrm{n}=5)$ \\
\hline Mouse 1 & 1.03 & 1.48 \\
\hline Mouse 2 & 1.3 & 1.59 \\
\hline Mouse 3 & 0.94 & 1.44 \\
\hline Mouse 4 & & 2.11 \\
\hline Mouse 5 & & 1.92 \\
\hline Mean \pm SEM & $1.09 \pm 0.1$ & $1.71 \pm 0.1$ \\
\hline
\end{tabular}

Table 1. Comparison of left ventricular (LV) wall thickness between healthy and experimental autoimmune myocarditis (EAM) mice. Three healthy and five EAM-induced mice were subjected to magnetic resonance microscopy (MRM) imaging on day 21 postimmunization. After acquiring the cardiac images by MRM, the thickness of LV wall was measured using Segment software as described in the protocol. The values displayed in the table represent LV wall thickness in $\mathrm{mm}$.

\section{Discussion}

This study describes the MRM procedure and its utility as a non-invasive tool to ascertain cardiac abnormalities in mice affected with autoimmune myocarditis. Since the histologic features of EAM resemble postinfectious myocarditis of humans, mouse models are commonly employed to delineate the immune mechanisms of myocardial injuries ${ }^{23-25}$. However, the animals affected with myocarditis appear clinically normal, and the diagnosis is made based on histology at the termination of experiments ${ }^{7}$. The animals are usually sacrificed on day 21 postimmunization. Assessing the disease process in this way at single time points limits use of these models, especially in pharmaceutical research, where monitoring of disease progression in response to treatments is a critical requirement.

To ascertain cardiac abnormalities in living animals, use of non-invasive modalities like MRM is helpful. The MRM technique that described here offers an advantage of obtaining the structural and functional characteristics of hearts without the need to use contrast agents. However, this technique requires acquisition of high resolution 3D anatomical images in strong magnetic fields. Nonetheless, once the images are acquired, functional parameters such as LV end-diastolic volumes and ejection fractions can be analyzed later using commercially available 
software, without the need to further assemble the MRM apparatus. As shown in Figure 2, MRM examination of animals immunized with Myhc- $\alpha$ 334-352 revealed LV wall thickness to be greater than in healthy mice (Figure 2B), with a corresponding decrease in functional outputs (LV enddiastolic volumes and ejection fractions; Figure 2C). As expected, hearts from immunized, but not healthy, animals had inflammatory infiltrates (Figure 2D). Thus, findings from the MRM technique and histology corroborate each other.

Nonetheless, to obtain reproducible results by MRM, the following three factors need to be addressed. (a) Animals should be placed in the MRM scanner so hearts are positioned in the center of the magnet to expose them to the magnetic field with maximum homogeneity. (b) Motion artifact is a concern in live animal experiments. To diminish image blurring due to respiration and cardiac movements, pulse oximetry and respirometry were used to gate MRM acquisition - that is, to acquire discrete image signals at specific time points within the respiratory and cardiac cycleswhich required the use of an animal monitoring system. (c) Acquisition of high-resolution 3D cardiac images is a critical requirement to enable detailed analysis of cardiac abnormalities. To obtain images in all three dimensions, and to increase signal-to-noise ratio, it is important to design more sensitive imaging coils specific to MRM that permit accurate and complete capturing of images in strong magnetic fields in a short-axis orientation within the animal scanners.

In conclusion, development of an MRM technique to evaluate cardiac abnormalities in live animals is challenging. This is especially true for mice, because of their smaller heart sizes ( 1/2,000 the mass of a human heart) and higher heart rates ( 600 beats per min) compared to humans ${ }^{26}$. Nonetheless, once developed and validated, the MRM technique can be used to compare the anatomical and functional changes of hearts between healthy and sick animals. Thus, the MRM technique could serve as a valuable, non-invasive tool to assess the longitudinal progression of inflammatory cardiac pathologies related to both the acute and chronic nature of the disease process, and to monitor responses to therapies in living animals.

\section{Disclosures}

No conflicts of interests were declared.

\section{Acknowledgements}

This work was supported by the National Institutes of Health (HL114669). CM is a recipient of a postdoctoral research fellowship grant awarded by the Myocarditis Foundation, NJ.

\section{References}

1. Heidenreich, P.A. et al. Forecasting the future of cardiovascular disease in the United States: a policy statement from the American Heart Association. Circulation. 123 (8), 933-944, doi: CIR.0b013e31820a55f5 [pii] 10.1161/CIR.0b013e31820a55f5, (2011).

2. Fujinami, R.S. et al. Molecular mimicry, bystander activation, or viral persistence: infections and autoimmune disease. Clin Microbiol Rev. 19 (1), 80-94, doi: 19/1/80 [pii] 10.1128/CMR.19.1.80-94, (2006).

3. Cihakova, D., \& Rose, N.R. Pathogenesis of myocarditis and dilated cardiomyopathy. Adv Immunol. 99, 95-114, doi: S0065-2776(08)00604-4 [pii] 10.1016/S0065-2776(08)00604-4, (2008).

4. Donermeyer, D.L. et al. Myocarditis-inducing epitope of myosin binds constitutively and stably to I-Ak on antigen-presenting cells in the heart. J Exp Med. 182 (5), 1291-1300, (1995).

5. Gangaplara, A. et al. Coxsackievirus B3 infection leads to the generation of cardiac myosin heavy chain-alpha-reactive CD4 T cells in A/J mice. Clin Immunol. 144 (3), 237-249, doi: S1521-6616(12)00166-0 [pii] 10.1016/j.clim.2012.07.003, (2012).

6. Huber, S.A., \& Lodge, P.A. Coxsackievirus B-3 myocarditis in Balb/c mice. Evidence for autoimmunity to myocyte antigens. Am J Pathol. 116 (1), 21-29, (1984).

7. Massilamany, C., et al. Identification of novel mimicry epitopes for cardiac myosin heavy chain-alpha that induce autoimmune myocarditis in A/J mice. Cell Immunol. 271 (2), 438-449, doi: S0008-8749(11)00214-0[pii] 10.1016/j.cellimm.2011.08.013, (2011).

8. Pummerer, C.L. et al. Identification of cardiac myosin peptides capable of inducing autoimmune myocarditis in BALB/c mice. J Clin Invest. 97 (9), 2057-2062, doi: 10.1172/JCl118642, (1996).

9. Rose, N.R., \& Hill, S.L. The pathogenesis of postinfectious myocarditis. Clin Immunol Immunopathol. 80 (3pt2), S92-99, doi: S0090122996901464 [pii], (1996).

10. Saraste, A. et al. Coronary flow reserve and heart failure in experimental coxsackievirus myocarditis. A transthoracic Doppler echocardiography study. Am J Physiol Heart Circ Physiol. 291, H871-875 DOI: 10.1152/ajpheart.01375.2005 (2006).

11. Altes, T.A. et al. Hyperpolarized $3 \mathrm{He}$ MR lung ventilation imaging in asthmatics: preliminary findings. J Magn Reson Imaging. 13 (3), $378-384$, doi: 10.1002/jmri.1054 [pii], (2001).

12. Driehuys, B. et al. Small animal imaging with magnetic resonance microscopy. ILAR J. 49 (1), 35-53, (2008).

13. Smith, B.R. Magnetic resonance microscopy with cardiovascular applications. Trends Cardiovasc Med. 6 (8), 247-254, doi: S1050-1738(96)00107-7[pii]10.1016/S1050-1738(96)00107-7, (1996).

14. Potter, K. Magnetic resonance microscopy approaches to molecular imaging: sensitivity vs. specificity. J Cell Biochem Suppl. 39, 147-153, doi: $10.1002 / j c b .10421,(2002)$.

15. Benveniste, H., \& Blackband, S. MR microscopy and high resolution small animal MRI: applications in neuroscience research. Prog Neurobiol. 67 (5), 393-420, doi: S0301008202000205 [pii], (2002).

16. Epstein, F.H. et al. MR tagging early after myocardial infarction in mice demonstrates contractile dysfunction in adjacent and remote regions. Magn Reson Med. 48 (2), 399-403, doi: 10.1002/mrm.10210, (2002).

17. Gewalt, S.L. et al. MR microscopy of the rat lung using projection reconstruction. Magn Reson Med. 29 (1), 99-106, (1993).

18. Kern, M.J. The cardiac catheterization handbook., Edn. 5th. (2011).

19. Heiberg, E. et al. Design and validation of Segment--freely available software for cardiovascular image analysis. BMC Med Imaging. 10, 1 , doi: 1471-2342-10-1 [pii] 10.1186/1471-2342-10-1, (2010). 
20. Cranney, G.B. et al. Left ventricular volume measurement using cardiac axis nuclear magnetic resonance imaging. Validation by calibrated ventricular angiography. Circulation. 82 (1), 154-163, (1990).

21. Hiba, B. et al. Cardiac and respiratory double self-gated cine MRI in the mouse at 7 T. Magn Reson Med. 55 (3), 506-513, doi: 10.1002/ mrm.20815, (2006).

22. Bryant, D. et al. Cardiac failure in transgenic mice with myocardial expression of tumor necrosis factor-alpha. Circulation. 97 (14), 1375-1381, (1998).

23. Neu, N. et al. Cardiac myosin-induced myocarditis as a model of postinfectious autoimmunity. Eur Heart J. 12 Suppl D, 117-120, (1991).

24. Neumann, D.A. et al. Induction of multiple heart autoantibodies in mice with coxsackievirus B3- and cardiac myosin-induced autoimmune myocarditis. J Immunol. 152 (1), 343-350, (1994).

25. Rose, N.R. et al. Postinfectious autoimmunity: two distinct phases of coxsackievirus B3-induced myocarditis. Ann N Y Acad Sci. 475, 146-156, (1986).

26. Farmer, J.B., \& Levy, G.P. A simple method for recording the electrocardiogram and heart rate from conscious animals. $\mathrm{Br} J \mathrm{Pharmacol}$ Chemother. 32 (1), 193-200, (1968). 